\title{
Brain-Wide Maps of Synaptic Input to Cortical Interneurons
}

\author{
CNicholas R. Wall, ${ }^{1,2}$ Mauricio De La Parra, ${ }^{1}$ Jordan M. Sorokin, ${ }^{1}$ Hiroki Taniguchi, ${ }^{3,4}$ Z. Josh Huang, ${ }^{4}$ \\ and 1 Edward M. Callaway ${ }^{1}$ \\ ${ }^{1}$ Systems Neurobiology Laboratories, Salk Institute for Biological Studies, La Jolla, California 92037, ${ }^{2}$ Neurosciences Graduate Program, University of \\ California, San Diego, La Jolla, California 92093, ${ }^{3}$ Max Planck Florida Institute for Neuroscience, Jupiter, Florida 33458, and ${ }^{4}$ Cold Spring Harbor \\ Laboratory, Cold Spring Harbor, New York 11724
}

Cortical inhibition is mediated by diverse inhibitory neuron types that can each play distinct roles in information processing by virtue of differences in their input sources, intrinsic properties, and innervation targets. Previous studies in brain slices have demonstrated considerable cell-type specificity in laminar sources of local inputs. In contrast, little is known about possible differences in distant inputs to different cortical interneuron types. We used the monosynaptic rabies virus system, in conjunction with mice expressing Cre recombinase in either parvalbumin-positive, somatostatin-positive (SST + ), or vasoactive intestinal peptide-positive (VIP + ) neurons, to map the brain-wide input to the three major nonoverlapping classes of interneurons in mouse somatosensory cortex. We discovered that all three classes of interneurons received considerable input from known cortical and thalamic input sources, as well as from probable cholinergic cells in the basal nucleus of Meynert. Despite their common input sources, these classes differed in the proportion of long-distance cortical inputs originating from deep versus superficial layers. Similar to their laminar differences in local input, VIP + neurons received inputs predominantly from deep layers while SST + neurons received mostly superficial inputs. These classes also differed in the amount of input they received. Cortical and thalamic inputs were greatest onto VIP + interneurons and smallest onto SST + neurons.

Key words: barrel cortex; cortex; inhibitory neuron; S1

\section{Significance Statement}

These results indicate that all three major interneuron classes in the barrel cortex integrate both feedforward and feedback information from throughout the brain to modulate the activity of the local cortical circuit. However, differences in laminar sources and magnitude of distant cortical input suggest differential contributions from cortical areas. More input to vasoactive intestinal peptide-positive (VIP+) neurons than to somatostatin-positive (SST + ) neurons suggests that disinhibition of the cortex via VIP + cells, which inhibit SST + cells, might be a general feature of long-distance corticocortical and thalamocortical circuits.

\section{Introduction}

Synaptic inhibition in the neocortex arises from diverse populations of GABAergic interneurons, which can be classified based

Received Nov. 3, 2015; revised Jan. 8, 2016; accepted Feb. 8, 2016.

Author contributions:N.R.W. and E.M.C. designed research;N.R.W.,M.D.L.P., and J.M.S. performed research;H.T. and Z.J.H. contributed unpublished reagents/analytic tools; N.R.W. and E.M.C. analyzed data; N.R.W. and E.M.C. wrote the paper.

This work was supported by the National Institutes of Health (EY022577, MH063912 to E.M.C. and MH07844 to Z.J.H.) and the Gatsby Charitable Foundation (to E.M.C.).

The authors declare no competing financial interests.

Correspondence should be addressed to Edward M. Callaway, Salk Institute for Biological Studies, Systems Neurobiology Laboratories, 10010 N. Torrey Pines Road, La Jolla, CA 92037. E-mail: callaway@salk.edu.

N. R. Wall's present address: Nancy Pritzker Laboratory, Department of Psychiatry and Behavioral Sciences, Stanford University School of Medicine, Stanford, CA 94304-5485.

M. De La Parra's present address: Department of Otolaryngology Head and Neck Surgery, University of California San Francisco, San Francisco, CA 94115.

J. M. Sorokin's present address: Neurosciences Graduate Program, Stanford Neurosciences Institute, Stanford University, Stanford, CA 94305 on their morphological, physiological, and biochemical properties (Fishell and Rudy, 2011). Although inhibitory interneurons only comprise $\sim 20 \%$ of cortical neurons, their effects on cortical computation are profound. Parvalbumin (PV)-expressing, fastspiking basket cells form the most numerous class of cortical interneuron, whose perisomatic, basket-shaped axonal boutons exert exquisite control over the spiking activity of connected neurons (Jones and Hendry, 1984; Tamás et al., 2000, 2004). Another major class of interneurons expresses the neuropeptide somatostatin (SST); the majority of SST-expressing interneurons are Martinotti cells, which target the distal dendritic compartments of pyramidal neurons (DeFelipe et al., 1989; DeFelipe and Jones, 1992; Kawaguchi and Kubota, 1997). In vivo studies implicate these cells in the preferential suppression of lateral cortical inputs 
(Adesnik et al., 2012; Nienborg et al., 2013). In mice, the final major, nonoverlapping class of interneurons in the neocortex expresses vasoactive intestinal peptide (VIP; Xu et al., 2010), and preferentially regulates the activity of other interneurons (Lee et al., 2013; Pfeffer et al., 2013). Recent in vivo studies have implicated these cells in cortical disinhibition via the inhibition of SST-positive (SST + ) neurons in several different cortical areas (Lee et al., 2013; Pi et al., 2013; Fu et al., 2014; Stryker, 2014; Zhang et al., 2014).

Previous in vitro studies have demonstrated highly specific local connections onto and from distinct inhibitory neuron types (Dantzker and Callaway, 2000; Caputi et al., 2009; Xu and Callaway, 2009; Pfeffer et al., 2013). For example, cell types that express VIP receive considerable local input from cortical layer 5, while SST interneurons receive little or no layer 5 input (Xu and Callaway, 2009). Understanding these circuits has guided detailed investigations of the functional impact of each cell type in vivo. Nevertheless, our understanding of cortical interneurons remains limited. Although the major sources of input to the barrel cortex are known, it is not clear whether all cortical inhibitory cell types receive significant innervation from each input brain area or whether there might be quantitative or qualitative differences in distant input to each cell type. To address these questions, we used the monosynaptic rabies virus (RV) system (Wickersham et al., 2007; Wall et al., 2010; Callaway and Luo, 2015), in conjunction with mice expressing Cre in genetically specified subsets of cortical interneurons (Hippenmeyer et al., 2005; Taniguchi et al., 2011), to elucidate the populations of neurons that innervate SST + , PV-positive $(\mathrm{PV}+)$, and VIP-positive $(\mathrm{VIP}+)$ interneurons. This approach has been used in more limited analyses of inputs onto inhibitory cell types in mouse primary visual cortex (V1; Fu et al., 2014; Zhang et al., 2014), and functional activation of projections from motor cortex to $\mathrm{S} 1$ cortex (Lee et al., 2013) or from cingulate cortex to V1 (Zhang et al., 2014) have both demonstrated much stronger long-distance corticocortical connections onto VIP + cells than onto SST+ interneurons.

We discovered that while all three interneuron types received similar proportions of cortical, thalamic, and cholinergic input, VIP + interneurons received more overall synaptic input from throughout the brain and they received their cortical input predominantly from deep layers, contrasting with the superficial layer input to SST + interneurons. These data demonstrate that all three classes of interneurons receive both feedforward and feedback information from diverse brain structures, and use this information to structure the activity of the local cortical column. Differences in the cortical cell types that provide input, as well as observed differences in input intensity, suggest that distant inputs contribute importantly to the unique functional role of each cell group.

\section{Materials and Methods}

All methods using live animals described below were in accordance with protocols approved by the Salk Institute Institutional Animal Care and Use Committee.

Virus production. Adeno-associated virus (AAV) serotype 8 expressing nuclear-localized eGFP (H2B-eGFP, "H") and rabies B19 glycoprotein (B19G, "B") in a Cre-dependent manner (AAV8-DIO-HB; https://www. addgene.org/37452) was produced via crude fractionation by the Salk Institute GT3 Core facility, and had titers of $3 \times 10^{12} \mathrm{IU} / \mathrm{ml}$. The AAV vector plasmid was produced by subcloning H2B-eGFP-F2A-B19G into the pAAV-DIO-ChR2-eYFP plasmid (courtesy of Karl Deisseroth, Stanford, CA; https://www.addgene.org/20298), via deletion of the TVA (“T”) sequence from pBOB-SynP-HTB (https://www.addgene.org/
30195), then replacing the ChR2-eYFP sequence with H2B-eGFP-F2aB19G. EnvA-pseudotyped, glycoprotein gene-deleted RV expressing mCherry (EnvA $+\mathrm{RV} d G-\mathrm{mCh}$ ) was produced as in Osakada and Callaway (2013) and had functional titers of $2 \times 10^{9} \mathrm{IU} / \mathrm{ml}$ as determined through infection of TVA-expressing HEK 293T cells.

Animal surgery and viral injection. PV-Cre (https://www.jax.org/ strain/8069; Hippenmeyer et al., 2005), SST-Cre (https://www.jax.org/ strain/13044; Taniguchi et al., 2011), and VIP-Cre (https://www.jax.org/ strain/10908; Taniguchi et al., 2011) mice were bred with Rosa26-LSLTVA mice (Seidler et al., 2008) to produce animals that expressed both Cre and TVA in a restricted subset of neurons in the mouse brain. Male and female adult mice ( $>6$ weeks old) were briefly induced under isoflurane and then injected with ketamine/xylazine $(100 \mathrm{mg} / \mathrm{kg}$ ketamine, 10 $\mathrm{mg} / \mathrm{kg}$ xylazine in sterile saline) to achieve stable anesthesia. Animals were then mounted in a rodent stereotax (David Kopf Instruments model 940 series) and head-fixed using ear bars, bite bar, and nose clamp. A small incision was made in the skin over the skull, exposing bregma, lambda, and the location of the desired injection site. A three-axis micromanipulator was used to measure spatial coordinates for bregma and lambda and verify proper skull positioning. A small drill hole was made in the skull, exposing the brain at the following coordinates: $0.75 \mathrm{~mm}$ caudal to bregma, $3.0 \mathrm{~mm}$ lateral to bregma. A glass injection pipette with internal tip diameter of $\sim 25 \mu \mathrm{m}$ was then lowered $0.50 \mathrm{~mm}$ ventral to the brain surface.

Mice received $90 \mathrm{nl}$ monohemispheric injections of AAV8-DIO-HB via pulsed pressure delivery (Picospritzer III, General Valve), at a rate of $\sim 3 \mathrm{~Hz}$ and $\sim 10 \mathrm{nl} / \mathrm{min}$. The injection pipette remained in the mouse brain for $5 \mathrm{~min}$ following viral injection to reduce backflow. Mice were then allowed to recover for 22-28 d before RV injection. EnvA $+\mathrm{RV} d G$-mCh was injected under the same conditions and injection volume as the initial AAV injection. RV was allowed to replicate and spread for $7 \mathrm{~d}$ before perfusion and tissue processing. For all experiments, age-matched and sex-matched C57BL/6 mice were used as controls.

Tissue processing. Animals were anesthetized with an overdose of ketamine/xylazine ( 250 and $25 \mathrm{mg} / \mathrm{kg}$, respectively), then intracardially perfused with $30 \mathrm{ml}$ solution containing $4 \%$ paraformaldehyde in $0.1 \mathrm{M}$ phosphate buffer, $\mathrm{pH}$ 7.2. After perfusion, the brain was isolated and transferred to a postfixative solution containing $4 \%$ paraformaldehyde and $30 \%$ sucrose in phosphate-buffered saline (PBS), and then incubated overnight at $4^{\circ} \mathrm{C}$ on a rotating shaker.

Forty-micrometer-thick coronal brain sections were prepared using a microtome with freezing stage, and tissue was separated into four groups to allow for multiple tissue manipulations. Tissue groups that were not used immediately were placed in a cryopreservative solution ( $30 \%$ glycerol, $30 \%$ ethylene glycol in PBS) and stored at $-20^{\circ} \mathrm{C}$.

Fixed tissue was immunostained using a standard protocol. To amplify GFP signal, we used a chicken polyclonal antibody raised against eGFP (1:500; Aves Labs), combined with a Cy2-conjugated anti-chicken secondary antibody (1:500; Jackson ImmunoResearch). To preserve mCherry signal, we used a rabbit polyclonal antibody against DsRed (1:250; Clontech) and amplified with a Cy3-conjugated anti-rabbit secondary antibody (1:500; Jackson ImmunoResearch). All tissue was counterstained with 4',6-diamidino-2-phenylindole (DAPI, $300 \mathrm{~nm}$ ) for 30 min to visualize cell nuclei. Immunostained tissue was mounted on chrome-gelatin subbed slides and allowed to dry overnight. Tissue was then dehydrated and defatted using a series of ethanol and xylenes immersion steps. Slides were then coverslipped using Krystalon (Harleco) mounting medium and glass coverslips.

Image acquisition and analysis. Slides were scanned on an Olympus VS120 semiautomated fluorescent slide scanner at $\sim 1 \mu \mathrm{m}$ resolution. Starter cells were manually identified by the coincident label of eGFP, DAPI, and mCherry. Starter cell location was determined relative to the pial surface using the Perpendicular line tool in Olympus VS-Desktop software, and the starter cell layer was manually determined by comparison with the DAPI counterstain. The locations of mCherry-expressing somata outside of the primary somatosensory cortex were manually identified by comparing both autofluorescence and the DAPI counterstain with the brain area borders defined in the Paxinos atlas. Every fourth section was analyzed in this manner for the PV-Cre and SST-Cre 

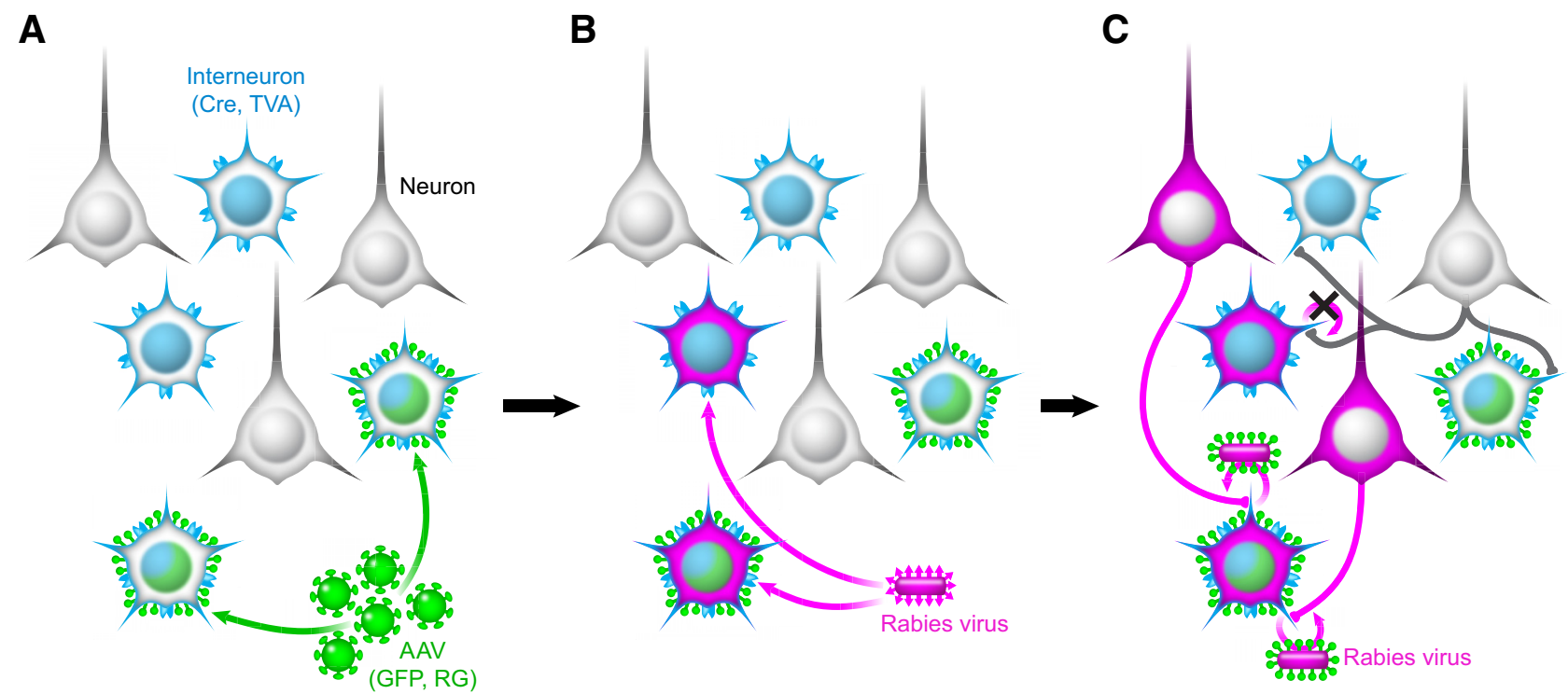

Figure 1. Monosynaptic RV system labels the direct presynaptic inputs to interneurons infected with both AAV and RV. A, Elements of the monosynaptic RV system. Rosa26-LSL-TVA mice were crossed with either PV-Cre, SST-Cre, or VIP-Cre animals to produce mice expressing Cre and TVA (light blue) in a restricted subset of neurons. AAV8-DI0-HB (green), when injected into cortex, expresses nuclear-localized eGFP and RG only in Cre-expressing interneurons. $\boldsymbol{B}$, EnvA $+\mathrm{RVdG}-\mathrm{mCh}$ (magenta) is injected in the same location 3-4 weeks later. This virus can only infect cells expressing TVA. C, Only cells that express both TVA and RG permit retrograde spread of RV. Thus, only cells expressing both H2B-eGFP (coexpressed with RG) and $\mathrm{mCherry} \mathrm{are} \mathrm{true} \mathrm{starter} \mathrm{cells.} \mathrm{One}$ week later, monosynaptic inputs to targeted interneurons are brightly labeled with mCherry.

mice. Every second section was analyzed for VIP-Cre mice due to the smaller number of starter cells in each animal.

\section{Results}

\section{Targeting specific classes of cortical interneurons}

To label the synaptic inputs to somatosensory cortical interneurons, Rosa26-LSL-TVA $\times$ interneuron-Cre mice were injected with $90 \mathrm{nl}$ of AAV8-DIO-HB (referred to as AAV or Credependent AAV), expressing nuclear-localized GFP and rabies glycoprotein (RG) in the barrel fields of primary somatosensory cortex (barrel cortex; Fig. 1A). The monosynaptic RV expressing mCherry (EnvA $+\mathrm{RVdG}-\mathrm{mCh}$ ) is pseudotyped with the avian sarcoma leucosis virus glycoprotein EnvA, and can only infect mammalian cells expressing the avian receptor TVA. This restricts RV infection to Cre-expressing interneurons of a single class. All interneurons of a specific type express Cre recombinase and TVA, but only a subset of these cells are infected with the Cre-dependent AAV (Fig. 1A). When RV is injected in the same location 3-4 weeks later, any neuron expressing TVA can be infected (Fig. 1B), but only rabies-infected cells expressing RG will permit the monosynaptic retrograde spread of the RV (Fig. $1 C)$. These neurons can be identified by the coincident expression of nuclear-localized GFP (from the Cre-dependent AAV) and mCherry (from the RV), and are hereafter referred to as "starter cells." Neurons directly presynaptic to starter cells will be labeled with mCherry expressed from RV and distributed throughout the brain; these neurons are observed 1 week after RV injection (Fig. 1C).

Figure 2 depicts the appearance of superficial layers of mouse barrel cortex in AAV + RV-infected SST-Cre (Fig. 2A), VIP-Cre (Fig. 2B), and PV-Cre (Fig. 2C) mice. mCherry, magenta; DAPI, blue. Scale bars, $50 \mu \mathrm{m}$. SST + interneurons are known to preferentially target the distal dendrites of pyramidal cells (Kawaguchi and Kubota, 1997), sending numerous projections into layer 1 and other superficial layers of the cortex, and can be seen in Figure $2 A$ (thin tangential fibers). In addition, distal dendrites from retrogradely infected pyramidal cells (thick radial fibers) are also immediately detected in layer 1 . Consistent with known VIP + interneuron morphology, the majority of RV-infected cells at the injection site of VIP-Cre mice are bipolar in nature (Fig. $2 B$ ), sending a primary neurite up to layer 1 , and another down to deeper layers of cortex. In contrast, layer 1 largely lacks $\mathrm{RV}$-labeled projections in PV-Cre mice, consistent with the known morphology of PV + cortical interneurons. Figure $2 D$ depicts the high concentration of basket-type synapses surrounding deeper-layer cortical neuronal somata in PV-Cre mice, which is consistent with the preponderance of $\mathrm{PV}+$ basket cells in this mouse line.

Figure 3 shows representative images of the virus injection site across all three interneuron-Cre lines, with higher-magnification inset areas in Figure $3 A-C$ indicated by dashed yellow boxes. Neurite labeling (RV, magenta) is visible in layer 1 of the SST-Cre animals (Fig. 3A), as expected from the distal dendritic-targeting population of SST + Martinotti cells. Starter cells (white dots) are distributed throughout layers 1-6. In the PV-Cre line, rabieslabeled starter interneurons are absent from layer 1 , as expected (Fig. 3B; Xu et al., 2010). In the VIP-Cre line, the morphology of rabies-infected neurons is strikingly different compared with the other two lines; many cells of the VIP + bipolar interneuron class are immediately visible, and starter cells are largely confined to the superficial cortical layers, consistent with the known distribution of VIP+ neurons in the mouse (Fig. 3C; Xu et al., 2010).

The spatial distribution of starter cells across all three interneuron types is illustrated in Figure 3D,E. SST + starter cells are marked in blue, $\mathrm{PV}+$ in red, and VIP + in orange. Figure $3 D$ charts the layer distribution of all starter cells for each interneuron class. SST + starter cells were found throughout the cortical layers with the highest proportions in L2/3, L5a, and L4 respectively. The proportion of layer $1-4$ SST + starter cells $(58 \%)$ is somewhat higher than the proportion found with SST antibody staining (48\%; Xu et al., 2010), and concordantly, the proportion of infragranular input ( 42 vs $52 \%$ ) is somewhat lower. This likely reflects a tendency for the virus injections in some animals to be 

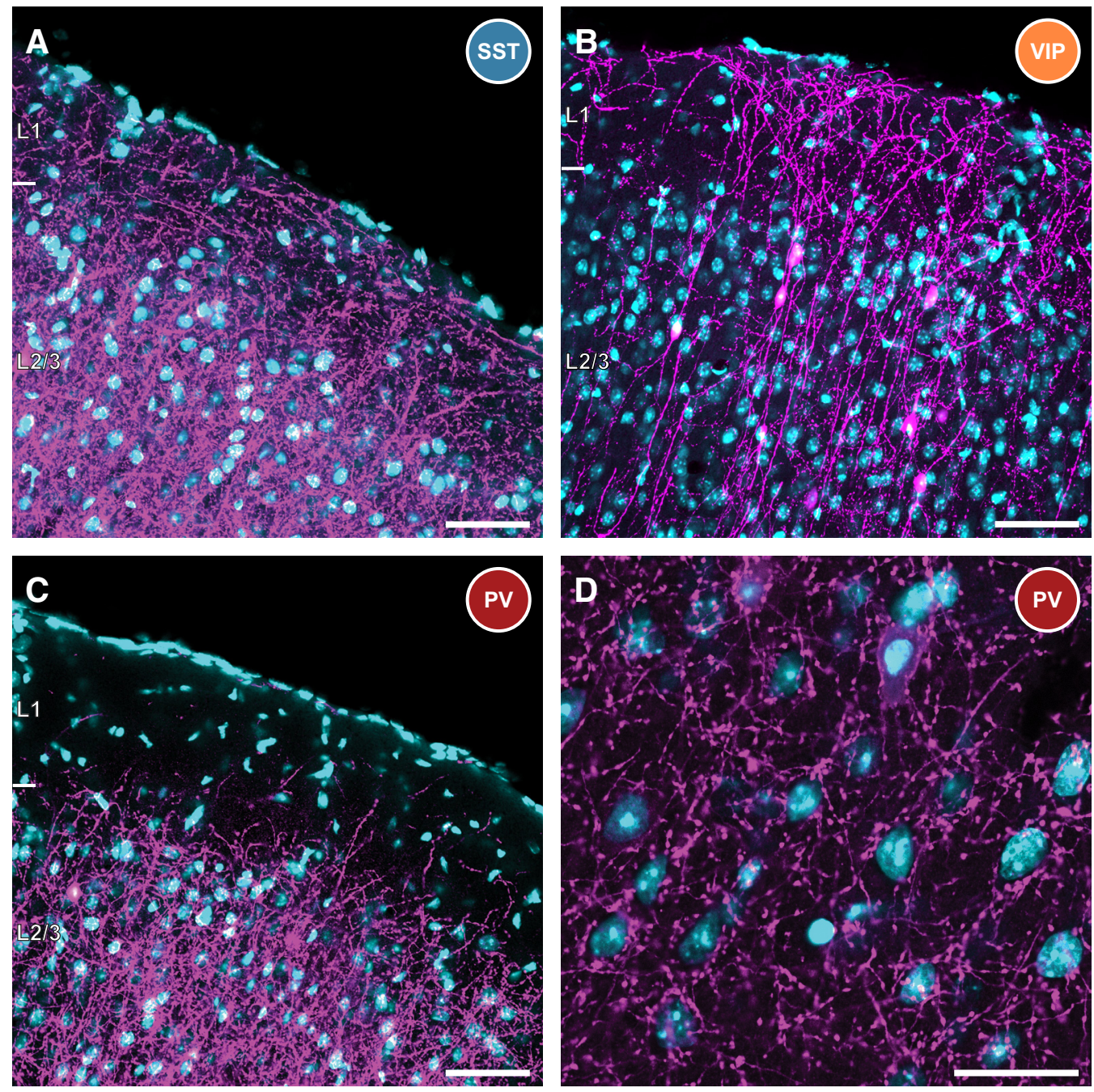

Figure 2. Characteristics of infected interneurons in SST, VIP, and PV-Cre mice. A, SST-expressing interneurons are known to heavily innervate the distal dendrites of cortical pyramidal cells. This is evidenced by the high density of projections in superficial layers, including high axon density in layer 1 . The distal dendrites of some retrogradely infected pyramidal cells are also visible extending into layer 1. RV, magenta; DAPI, cyan. This is consistent for all panels. Scale bar, $50 \mu \mathrm{m}$. B, Rabies-infected VIP-expressing interneurons are largely bipolar in nature, with a primary neurite extending into layer 1, and the other projecting to deeper layers of cortex. The cell bodies of these neurons are primarily found in superficial cortical layers. Scale bar, $50 \mu \mathrm{m}$. $C$, As expected from the properties of PV + interneurons, very few projections are detected in layer 1 of PV-Cre mice following AAV and RV infection. However, there is heavy innervation of deeper cortical layers. Scale bar, $50 \mu \mathrm{m}$. $\boldsymbol{D}$, Fluorescently labeled basket-type synapses are prevalent in PV-Cre mice, ringing neuronal somata in deeper layers of cortex. This is consistent with efficient targeting to PV-expressing basket cells in this mouse line. Scale bar, $25 \mu \mathrm{m}$.

biased toward superficial layers. As expected, PV+ starter cells are completely absent from layer 1 , and are distributed similarly to the native density of PV+ interneurons described in $\mathrm{Xu}$ et al. (2010). VIP + starter cells were primarily detected in the superficial and granular layers of cortex. This layer pattern is also largely consistent with the previously described distribution of VIP+ interneurons (Xu et al., 2010). Figure $3 E$ charts the distribution of starter cells relative to the pial surface, with each column indicating one animal. One animal each from the PV-Cre and VIP-Cre cohort lacked detectable starter cells and were excluded from analysis.

\section{Long-range inputs to cortical interneurons}

Inputs to 418 starter interneurons in 11 mice (SST-Cre), 247 neurons in 7 mice (PV-Cre), and 61 neurons across 7 mice (VIP-Cre) were quantified, and the proportions of brain-wide inputs to all three major cortical interneuron classes are depicted in Figure 4A. Each bar indicates the mean proportion of total input that arose from a single brain structure, weighted equally across each animal within a group. The thin line indicates SEM. The ipsilateral primary somatosensory cortex contained large numbers of input cells, but was excluded from analysis due to the mixed population of directly rabies-infected interneurons $\left(\mathrm{TVA}+, \mathrm{RG}^{-}\right)$and input cells at the injection site. Data are displayed for 14 different brain areas; only brain regions that possessed mCherry-labeled neurons in $\geq 3$ animals across all groups are displayed independently. Some cortical areas were clustered to reach this threshold; auditory cortices include both the primary and the secondary auditory cortices. Limbic cortices include inputs from the perirhinal cortex, the ectorhinal cortex, the retrosplenial cortex, the anterior cingulate cortex, and the lateral entorhinal cortex. Any remaining long-range input neurons were clustered into the appropriate "other" groups.

\section{Similar proportion of long-range cortical input to all interneuron types}

Major long-range cortical input sources to cortical interneurons included the secondary somatosensory cortex (S2), the primary 

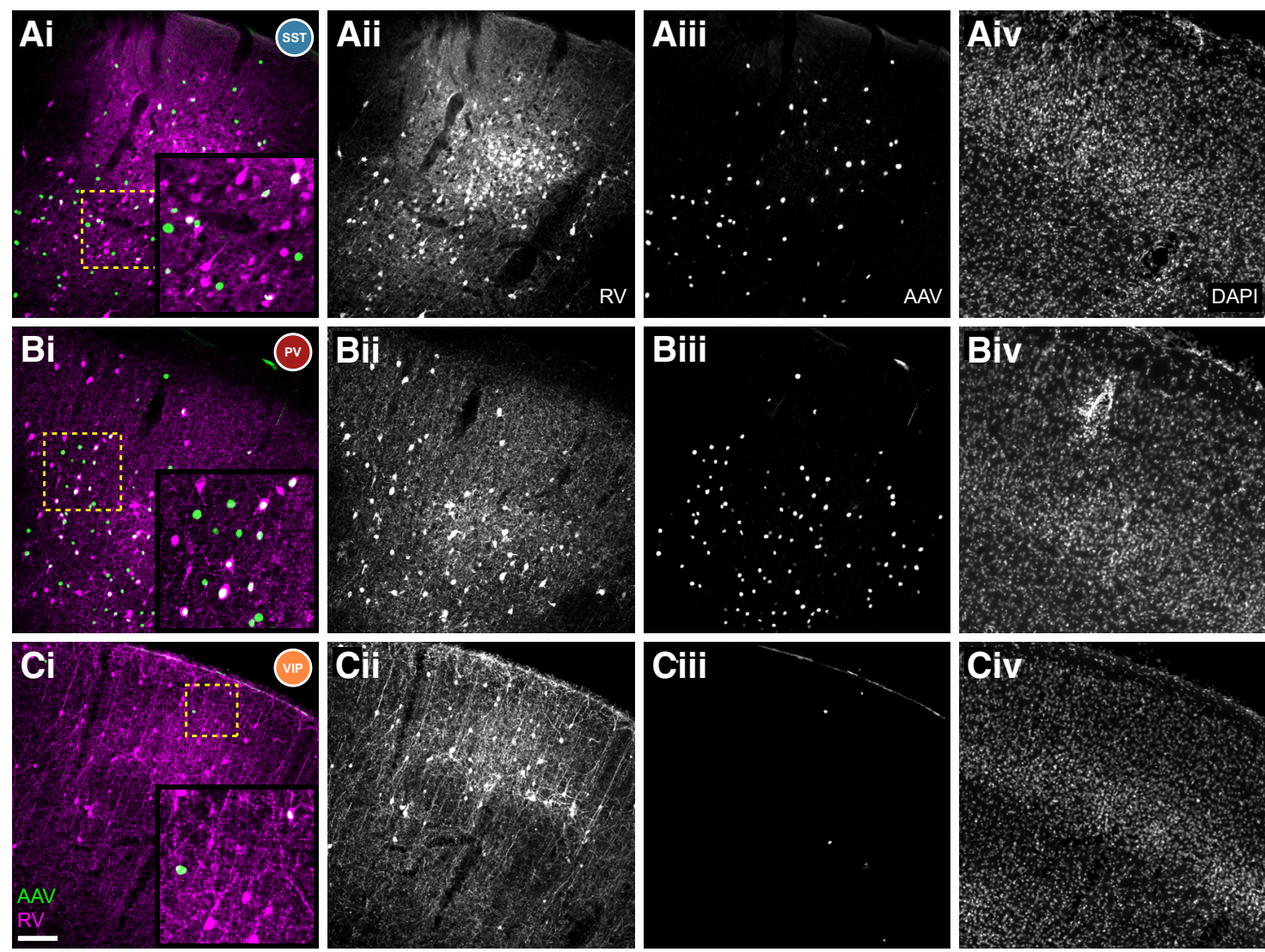

D SST

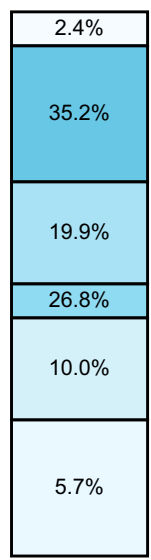

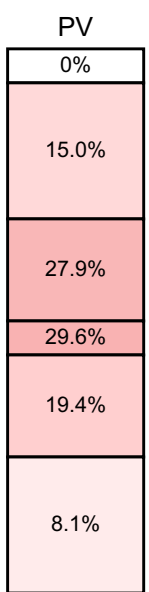

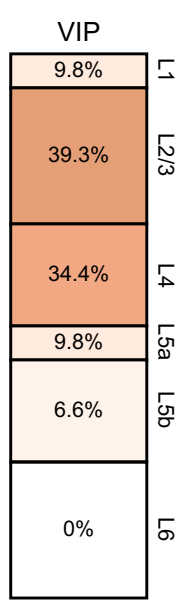

E

Starter cell type

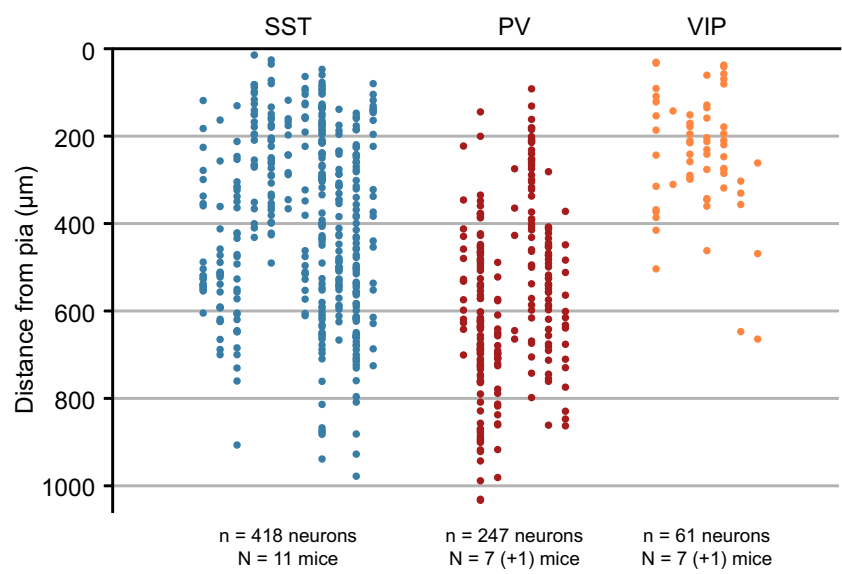

Figure 3. Population description of targeted interneurons in the barrel cortex. $A_{i}$ Barrel cortex in SST-Cre mice. AAV-infected (green) and RV-infected (magenta) cells are numerous, and starter cells (white) are found throughout cortical layers. As expected, SST + interneurons and fluorescent neurites are detectable in layer $1 . A_{i i}-C_{i v}$, Single fluorescence channels for RV $\left(A_{i j}\right), \operatorname{AAV}\left(A_{i i i}\right)$, and $\operatorname{DAPI}\left(\boldsymbol{A}_{i v}\right)$ are also included. $\boldsymbol{B}_{i i}-\boldsymbol{B}_{i v}, \boldsymbol{C}_{i i}-\boldsymbol{C}_{i v}$, Single fluorescence channels are labeled as in $\boldsymbol{A}_{i j}-\boldsymbol{A}_{i v}$. Scale bar: (in $\left.\boldsymbol{C}_{i}\right), \boldsymbol{A}_{i}-\boldsymbol{C}_{i v}, 100 \mu \mathrm{m}$. $\boldsymbol{A}_{i,} \boldsymbol{B}_{i,} \boldsymbol{C}_{i}$, Higher-magnification insets are indicated by dashed yellow boxes. $\boldsymbol{B}$, Barrel cortex in PV-Cre mice. As expected, starter cells can be found in layers $2-6$ but are absent from layer 1 . Neurite label is absent in layer 1 , but basket-type bouton clusters are detectable in deeper layers, as expected. C, Barrel cortex in VIP-Cre mice. Relatively few starter cells are detected, but are most prevalent in superficial layers. As expected, interneuron morphology is largely bipolar in nature. D, Layer distribution of starter interneurons. Most SST + starter cells were found in layers $2 / 3$ and $5 \mathrm{a}$, as expected from targeting a population enriched with Martinotti cells. PV + starter cells were distributed throughout layers $2-6$, but never in layer 1. VIP + starter cells were largely contained in superficial cortical layers. $E$, Individual distribution of starter interneurons. Each column indicates one animal, and the $y$-axis indicates starter cell distance from pia. Each filled circle indicates a single starter cell.

motor cortex (M1), and the contralateral primary somatosensory cortex. These brain regions are known to be the primary sources of long-range cortical information to the barrel cortex. Minor inputs were detected from auditory, limbic, and secondary visual cortices, with a small number of neurons from other cortical structures. When normalized so that each mouse in a cohort was equally weighted in population averages, long-range corticocortical inputs comprised the following percentage of total input:
SST-Cre, $41.0 \pm 8.3 \%$; PV-Cre, $48.4 \pm 6.1 \%$; VIP-Cre, $50.3 \pm$ $9.0 \%$ (mean \pm 1 SEM).

Overall, each interneuron class received similar proportions of input from all major connected cortical input structures $(p>0.05$ for each individual cortical region via one-way ANOVA). Representative images of neuronal inputs from S2 (Fig. 4B, right dotted box) are shown for SST-Cre (Fig. 4C), PV-Cre (Fig. $4 D$ ), and VIP-Cre (Fig. $4 E$ ) mice. To better iden- 

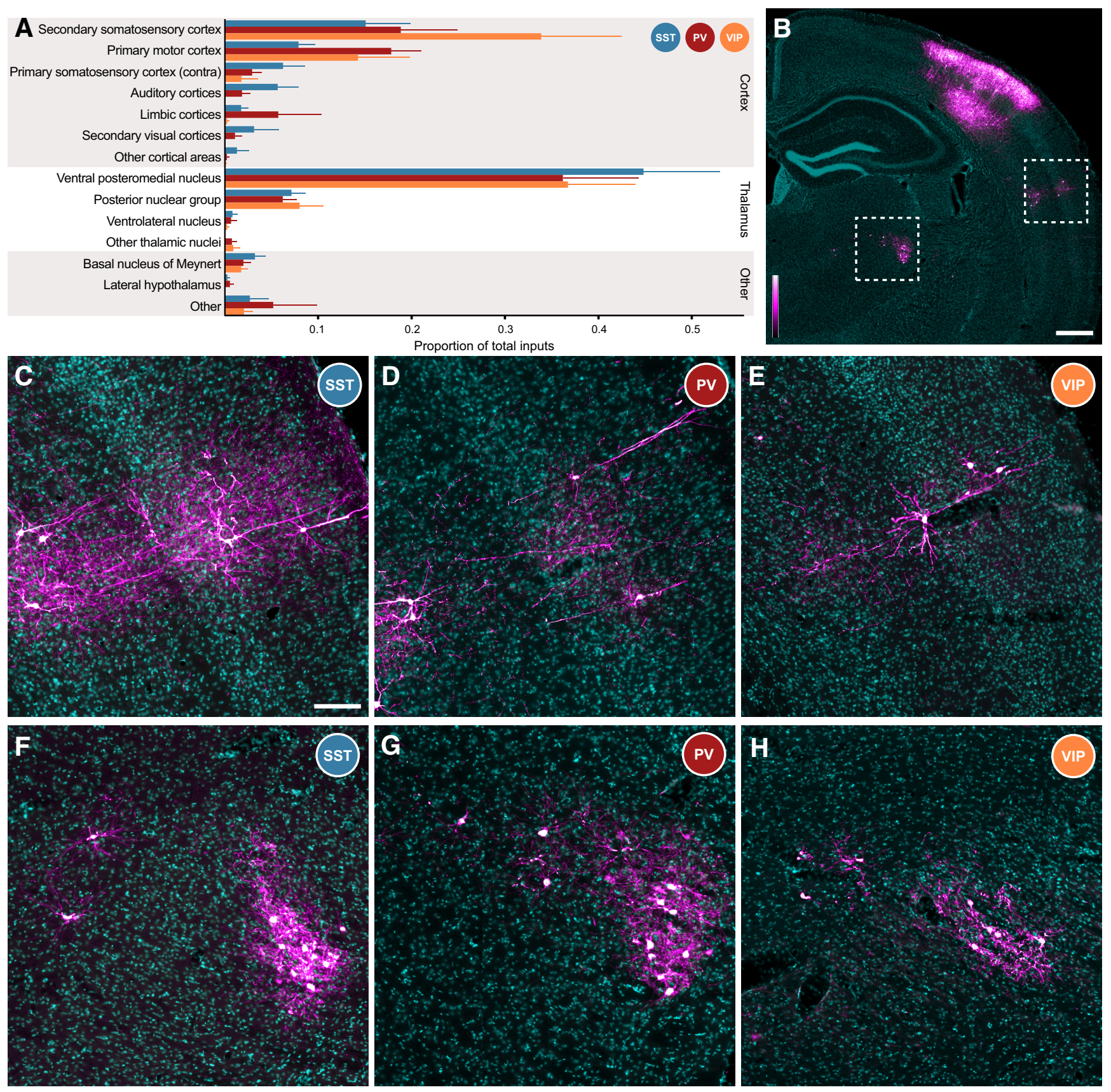

Figure 4. Distribution of brain-wide inputs to cortical interneurons. A, Overall, proportional input to all three interneuron classes was similar. All three interneuron types received inputs from secondary somatosensory cortex (S2), primary motor cortex (M1), and contralateral primary somatosensory cortex (S1BF), the major sources of long-range corticocortical input to S1. All three classes also received similar proportions of input from the VPm and the $\mathrm{P}$, the primary and secondary thalamic nuclei known to project into $\mathrm{S} 1$. Other minor input streams, such as innervation from the basal nucleus of Meynert, were also detected. Thick bars depict mean proportion of inputs, and thin lines indicate +1 SEM. $\boldsymbol{B}$, Low-magnification depiction of a brain slice from a PV-Cre mouse. S2 is indicated by the dashed box at the far right, and is depicted at higher magnification for all three mouse lines in $\boldsymbol{C}-\boldsymbol{E}$. A region of thalamus, including nuclei VPm and $P_{0}$, is outlined by the dashed box to the left, and is explored at higher magnification in $\boldsymbol{F}-\boldsymbol{H}$. RV signal is depicted with a custom magenta-hot lookup table (see lookup table to bottom left), and DAPl is in cyan. This is consistent across all panels. Scale bar, $500 \mu \mathrm{m}$. C-E, S2 inputs to $\mathbf{S 1}$ interneurons are largely detected in deep layer 3, layer $5 \mathrm{~A}$, and along the border between layers $5 \mathrm{~B}$ and 6 . Although in the images depicted cortical inputs are most numerous in the SST-Cre line (C), the overall proportion of inputs is similar across the PV-Cre (D) and VIP-Cre (E) lines. Scale bar: (in $\boldsymbol{C}) \boldsymbol{C}-\boldsymbol{H}, 100 \mu \mathrm{m}$. $\boldsymbol{F}-\boldsymbol{H}$, Thalamic inputs to S1 interneurons are present in both Po (left) and VPm (right), with many more cells within the boundaries of VPm. Again, total inputs depicted to SST (F) interneurons are most numerous, but the overall proportion of inputs is consistent across the PV-Cre $(\boldsymbol{G})$ and VIP-Cre $(\boldsymbol{H})$ lines.

tify neuronal morphology, a custom magenta-hot lookup table is used in Figure $4 B-H$ to show RV labeling (Fig. 4B). Many input neurons in S2 were found either in deep layer 3, layer $5 \mathrm{~A}$, or at the border of layers 5B and 6, with significant input from remaining layers. Input neurons were present in layers $2-6$ of other cortical input areas. Although the total number of labeled neurons tended to be higher in SST-Cre mice, these mice also tended to have the largest number of starter cells (Fig. 3E), leading to similar proportions of input from cortical structures across all three mouse lines. Although input neurons in the auditory and secondary visual cortices were not detected in VIP-Cre mice, this is likely due to the small number of starter cells combined with the relatively small contribution of these regions to the innervation of the barrel cortex. 


\section{Similar proportion of thalamic, other input to each interneuron type}

Thalamic projection neurons provided the other major longrange source of synaptic input to cortical interneurons (SST-Cre, $52.8 \pm 8.8 \%$; PV-Cre, $43.8 \pm 9.1 \%$; VIP-Cre, $45.9 \pm 8.3 \%$; mean \pm 1 SEM). The single largest source of input to all three interneuron types arose from the ventral posteromedial nucleus of the thalamus (VPm), with other major input arising from the posterior nuclear group (Po; Fig. 4A). There is also a small amount of unexpected input from the ventral lateral nucleus of the thalamus. This could be indicative of a small number of starter cells originating outside the barrel cortex; however, no such starter neurons were ever detected. DAPI counterstain provides incomplete information regarding thalamic nuclear borders, so thalamic input neurons were primarily classified based on their stereotaxic coordinates. Since the ventral lateral nucleus of the thalamus is directly adjacent to both the VPm and the Po, it is possible that a small number of neurons were mislabeled and actually belonged to the VPm/Po thalamic group.

Representative images from the thalamus are depicted in Figure $4 F-H$, with the overall region indicated by the leftward dashed box in Figure $4 B$ (Po, left; VPm, right). Again, although the total number of inputs varied across mouse lines, proportional input from each nucleus was similar across classes $(p>$ 0.05 for each thalamic group via one-way ANOVA).

Other brain structures provided a relatively small amount of input (SST-Cre, $6.1 \pm 2.1 \%$; PV-Cre, $7.7 \pm 4.4 \%$; VIP-Cre, $3.8 \pm$ $1.1 \%)$. Of note, putative cholinergic neurons in the basal nucleus of Meynert provided direct synaptic input to all three classes of interneurons. These data indicate that all three major interneuron classes sample information from projection cells residing in many regions throughout the brain, and that there does not appear to be a strong proportional input bias toward any single cell type.

\section{VIP+ interneurons receive more input per starter cell}

Although all three types of interneurons received similar proportions of input from connected brain structures, it is possible that the absolute input levels onto each cell type could be different. To examine this possibility, we calculated the number of input neurons per starter cell for each animal across all three interneuron groups for the 14 regions defined in Figure 4. These data are plotted in Figure $5 A$; each bar represents the mean number of inputs per starter cell for all animals within a group, and the thin line indicates the SEM.

We then produced a metric of overall input magnitude onto each interneuron class, which we defined as the $\log [$ ( total input cells +1$) /($ total starter cells +1$)]$. These values were calculated for each animal, and are plotted in Figure $5 B$. The shaded range represents the two middle quartiles, and the horizontal bar contained within indicates the median value for each group. Whiskers indicate the full range of data for each group. Since the input-cell to starter-cell ratio for each animal is $\sim 1$, group data will be log-normally distributed rather than normally distributed (in particular, PV-Cre input-cell/starter-cell ratio demonstrably deviates from normality; $W=0.74, p<0.05$ via Shapiro-Wilk normality test). The small additions to both input cell and starter cell count reduce the effects of small number bias on the population description. Input magnitude onto the VIP interneuron population was significantly greater compared with the SST population (population means for all three classes different by oneway ANOVA, $F_{(2,22)}=5.95, p=0.009$, VIP vs SST $p<0.01$ via Tukey's test).
To determine the origin of the greater input onto VIP + interneurons, we then compared the amounts of inputs arising from cortex and thalamus. Cortical input magnitude, as defined previously, was summed across all cortical input regions, and was greater onto VIP interneurons compared with the SST population (Fig. $5 C$, three population means different via one-way ANOVA, $F_{(2,22)}=10.87, p=5 \times 10^{-4}$; VIP vs SST $p<0.01$ via Tukey's test). Thalamic input magnitude is diagrammed in Figure $5 D$; although VIP + interneurons tended to receive greater thalamic input magnitude, variability across animals prevented this observation from reaching significance (one-way ANOVA, $\left.F_{(2,22)}=2.97, p=0.07\right)$.

Together, these observations suggest that although all interneuron classes receive proportionally similar input from various brain regions, individual VIP + interneurons receive greater numbers of inputs than individual interneurons of other classes. Since VIP+ interneurons preferentially innervate other inhibitory interneurons, this could indicate a potentially profound source of disinhibition in the local cortical circuit.

\section{VIP+ interneurons receive more deep-layer cortical input}

Since we observed that VIP + interneurons received greater cortical input magnitude than the other neuronal classes, and studies of local connectivity in mouse S1 demonstrate a higher proportion of excitatory input from layer 5 onto VIP + neurons than onto SST + neurons (Xu and Callaway, 2009), we examined the layer distribution of long-range cortical inputs in greater detail. We separated cortical inputs into superficial (layer 2-3) and deep (layer 5-6) populations, and observed that VIP + interneurons received a greater proportion of deep-layer inputs (Fig. $5 E$, three population means different via one-way ANOVA, $F_{(2,22)}=5.85$, $p=0.009$; VIP vs SST $p<0.01$ via Tukey's test). A small population of inputs was found encroaching upon the border between layers 3 and 4, particularly in the secondary somatosensory cortex; these are clustered into the superficial layer input group. These neurons could be displaced deep layer 3 cells, or could represent an independent population of projection neurons.

We then performed input magnitude analysis on the superficial and deep-layer cortical inputs to the three interneuron types. As expected, deep-layer cortical input magnitude to VIP+ interneurons was greater than onto the other two interneuron groups (Fig. $5 G$, three population means different via one-way ANOVA, $F_{(2,22)}=14.97, p=8 \times 10^{-5}$; VIP vs SST $p<0.01$, VIP vs $\mathrm{PV} p<0.05$, PV vs SST $p<0.05$ via Tukey's test). Interestingly, even though the proportion of superficial input to VIP + interneurons was the lowest of the three populations, superficial layer cortical input magnitude was still greatest onto VIP+ interneurons (Fig. $5 F$, three population means different via oneway ANOVA, $F_{(2,22)}=4.01, p=0.03$; VIP vs SST $p=0.03$ via Tukey's test), further indicating that VIP+ interneurons receive the most overall cortical input, as seen in Figure $5 C$.

\section{Discussion}

By studying the brain-wide sources of monosynaptic input to $\mathrm{VIP}+, \mathrm{PV}+$, and SST + neurons in the S1 cortex, we have demonstrated that all three groups of interneurons receive inputs in similar proportions from the known cortical and subcortical structures that connect to S1. Nevertheless, the inputs to these different interneuron populations differ in two important respects. VIP + neurons receive a significantly higher proportion of their distant cortical inputs from the deep cortical layers than do $\mathrm{PV}+$ or SST + neurons. Furthermore, the magnitude of distant cortical inputs onto VIP + neurons is significantly greater than 

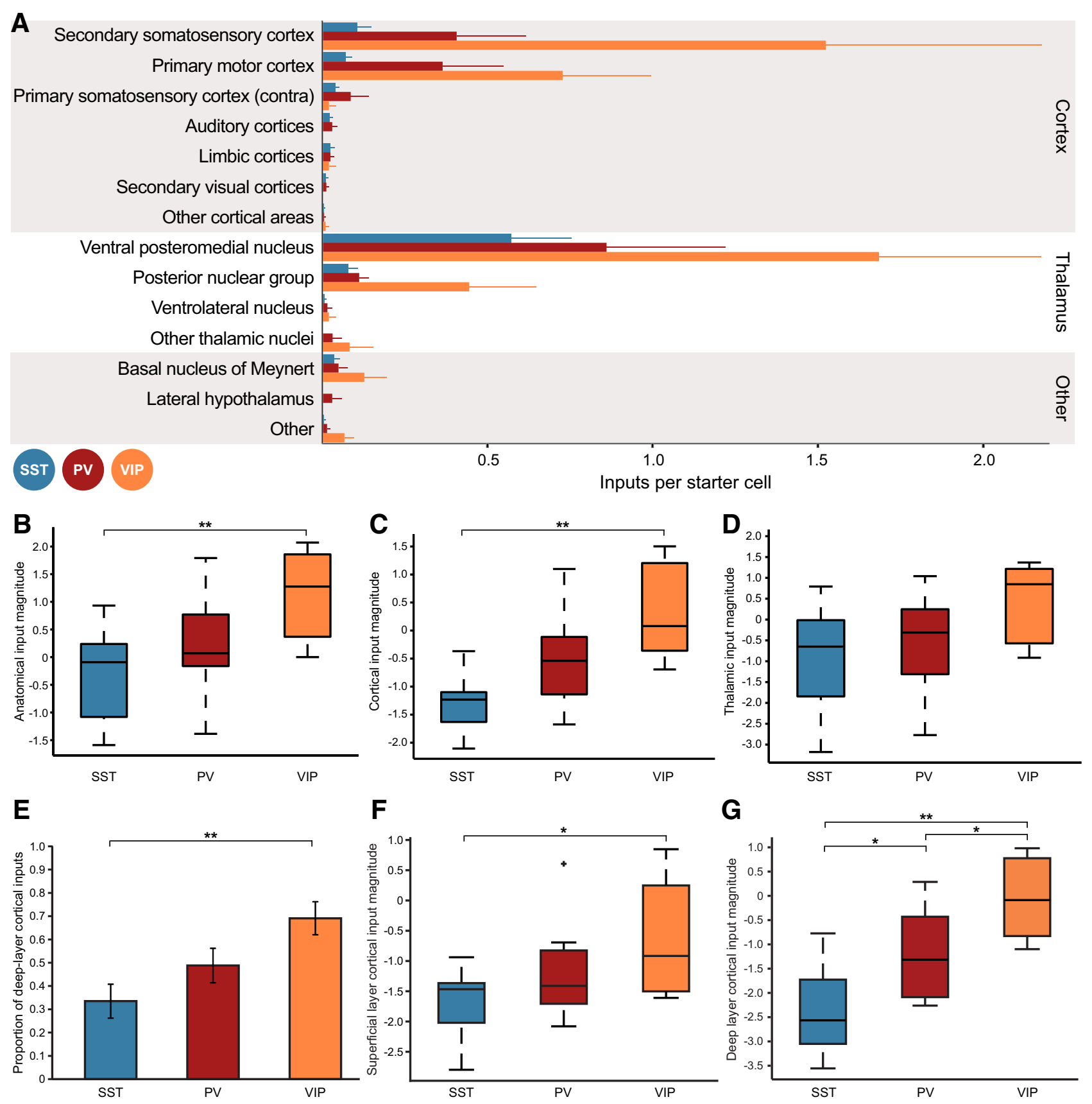

Figure 5. Number of input neurons per starter cell varies across cortical interneuron class. $A$, Plot of number of input neurons per starter cell for each of the brain regions described in Figure $4 A$. $\mathrm{VIP}+$ interneurons tend to receive greater numbers of inputs per starter cell than other interneuron types; this is quantified in $\boldsymbol{B}-\boldsymbol{D}$. Thick bars depict mean numbers of inputs per starter cell, and the thin line indicates +1SEM. $\boldsymbol{B}$, Anatomical input magnitude distribution for each interneuron class. Input magnitude is defined as the natural log of the number of input neurons per starter cell, with a small modifier for division of small numbers (see main text). Colored boxes indicate the $25^{\text {th }}-75^{\text {th }}$ percentiles. The horizontal line contained in each colored box indicates the median input magnitude for that interneuron class. Whiskers indicate the full extent of the data. When summed across all brain areas, SST input is weaker than VIP input $(p<0.01)$, with PV input magnitude tending to fall between the other two interneuron types. C, Cortical input magnitude distribution is calculated as in $\boldsymbol{B}$, but only for cortical inputs. Again, cortical input to SST cells is weaker than that to VIP interneurons $(p<0.01)$. D, Thalamic input magnitude distribution. Although there was a tendency toward a difference between the three groups ( $p=0.07$ by one-way ANOVA), variability across mice prevented any post hoc comparison. $\boldsymbol{E}$, Proportion of deep layer (layer 5-6) input compared with total cortical input. VIP interneurons receive a greater proportion of deep-layer input than SST interneurons $(p<0.01)$. $\boldsymbol{F}$, Superficial layer (layer $2-3)$ cortical input magnitude. Even though VIP + interneurons received the smallest proportion of superficial layer inputs (Fig. 5E), VIP + interneurons still received higher levels of superficial input per starter cell than SST + interneurons $(p<0.05)$. A single outlier $(>2.7 \sigma)$ in the PV interneuron group is plotted as a black cross. $\boldsymbol{G}$, Deep layer (layer 5-6) cortical input magnitude. As expected from C and $\boldsymbol{E}$, VIP interneurons have the greatest deep-layer input strength, followed by PV interneurons, then finally the SST cell population (VIP vs PV, $p<0.05 ;$ VIP vs SST, $p<0.01 ;$ PV vs SST, $p<0.05$ ).

onto SST + neurons. Thalamic inputs were also greater onto VIP+ neurons than SST+ neurons, but the difference did not reach statistical significance. These observations, along with previously published results, have important implications for the functional roles of each inhibitory cell group.
Since mammalian neural networks are typically composed of large numbers of cell types that are intimately intertwined through both direct and indirect connections, it is difficult to predict functional interactions from static wiring diagrams. Nevertheless, the organizational features of network connectivity can 
aid in the generation of hypotheses to be tested by measuring or manipulating the activity of specific network components. Here we have identified differences in input magnitude to two different inhibitory cell classes, VIP + and SST + neurons. These cell classes are known to directly and strongly interact with each other via local, biased connections from VIP + neurons onto SST+ neurons (Lee et al., 2013; Pfeffer et al., 2013). Thus, although these cell types receive similar proportionate input from distant structures, observed differences in input magnitude suggest likely impacts on cortical processing. In particular, the observation that greater input to VIP+ neurons is a feature of cortical inputs, and possibly also thalamic inputs, predicts that these input sources will activate VIP + neurons more strongly than SST + neurons, allowing VIP + neurons to strongly inhibit SST + cells without having to overcome excitatory inputs.

As monosynaptic RV tracing becomes more widely adopted, increasing evidence is mounting to suggest that there are physiological correlates to observed monosynaptic rabies input patterns. However, since rabies tracing is an anatomical measure, the functional impact of the differences in input magnitude that we have observed cannot be directly inferred without further study. Nevertheless, greater cortical input onto VIP+ neurons has been directly demonstrated functionally for the M1 to S1 (Lee et al., 2013) and the cingulate cortex to V1 pathways (Zhang et al., 2014), and our results suggest that this circuit mechanism is likely to generalize to other distant input sources. A recent functional study in the mouse corticostriatal system has also demonstrated the presence of a unique electrophysiological input stream from the somatosensory cortex onto direct pathway medium spiny neurons in the dorsal striatum (Sippy et al., 2015), a result originally observed using monosynaptic RV tracing (Wall et al., 2013).

Although we did not observe differences between inhibitory cell types in the proportion of inputs that they receive from distant structures, we did find that VIP + interneurons receive a significantly greater proportion of their distant cortical input from deep layers than do SST + interneurons. This observation is reminiscent of observed differences in local excitatory input sources onto different types of layer $2 / 3$ inhibitory neurons in mouse S1. SST + inhibitory neurons receive very little excitatory input from deep cortical layer 5, while cell types that are likely to include VIP + inhibitory neurons receive strong layer 5 input (Xu and Callaway, 2009). While it is not known what in vivo conditions might recruit cortical inputs originating from layer 5 versus more superficial layers, anatomical evidence indicates that layer 5 projections are likely to be more diffuse and less focused than those from layer 2/3 (Markov et al., 2014). This difference might therefore contribute to the ability of feedback cortical connections to mediate center-surround interactions inferred from differential effects of cingulate cortex activation on VIP + versus SST + neurons in V1 (Zhang et al., 2014).

It is important to note that all three interneuron cell classes that we have studied here $(\mathrm{SST}+, \mathrm{PV}+$ and VIP +$)$ are likely to be composed of multiple cell types. Previous in vitro studies that have focused on the connectivity of individual neurons identified more clearly distinguished cell types, and have revealed more distinct differences in local input sources than in the brain-wide patterns that we observed. We therefore expect that future studies that further separate these cell types might find more distinct differences in input sources than we have identified here. Such differences include not only distinctions based on morphology, intrinsic physiology, and gene expression (e.g., fast-spiking PV+ basket cells vs SST + Martinotti cells), but also differences in the laminar location of neurons of otherwise similar types (e.g., Martinotti cells in layer $2 / 3$ vs layer 5 ).

It is also noteworthy that VIP + inhibitory cortical neurons are likely to be composed of an even more diverse population of cell types than SST + or PV + neurons (Xu and Callaway, 2009; Lee et al., 2010; Miyoshi et al., 2010; Xu et al., 2010). Notably, only about half of VIP + neurons in mouse $S 1$ cortex express calretinin (CR; Lee et al., 2010) and these are likely to be largely responsible for the inhibition of SST + neurons observed from the VIP+ population (Meskenaite, 1997; Caputi et al., 2009). CR-positive and CR-negative VIP + cells also differ in their intrinsic firing properties (Lee et al., 2010; Miyoshi et al., 2010). It would therefore not be surprising if there were also differences in the sources and/or strength of input to these different cell populations. Future studies might explore these differences using intersectional genetic strategies along with VIP-Flp and CR-Cre mouse lines.

\section{References}

Adesnik H, Bruns W, Taniguchi H, Huang ZJ, Scanziani M (2012) A neural circuit for spatial summation in visual cortex. Nature 490:226-231. CrossRef Medline

Callaway EM, Luo L (2015) Monosynaptic circuit tracing with glycoprotein-deleted rabies viruses. J Neurosci 35:8979-8985. CrossRef Medline

Caputi A, Rozov A, Blatow M, Monyer H (2009) Two calretinin-positive GABAergic cell types in layer $2 / 3$ of the mouse neocortex provide different forms of inhibition. Cereb Cortex 19:1345-1359. CrossRef Medline

Dantzker JL, Callaway EM (2000) Laminar sources of synaptic input to cortical inhibitory interneurons and pyramidal neurons. Nat Neurosci 3:701-707. CrossRef Medline

DeFelipe J, Jones EG (1992) High-resolution light and electron microscopic immunocytochemistry of colocalized GABA and calbindin D-28k in somata and double bouquet cell axons of monkey somatosensory cortex. Eur J Neurosci 4:46-60. CrossRef Medline

DeFelipe J, Hendry SH, Jones EG (1989) Synapses of double bouquet cells in monkey cerebral cortex visualized by calbindin immunoreactivity. Brain Res 503:49-54. CrossRef Medline

Fishell G, Rudy B (2011) Mechanisms of inhibition within the telencephalon: "where the wild things are." Annu Rev Neurosci 34:535-567. CrossRef Medline

Fu Y, Tucciarone JM, Espinosa JS, Sheng N, Darcy DP, Nicoll RA, Huang ZJ, Stryker MP (2014) A cortical circuit for gain control by behavioral state. Cell 156:1139-1152. CrossRef Medline

Hippenmeyer S, Vrieseling E, Sigrist M, Portmann T, Laengle C, Ladle DR, Arber S (2005) A developmental switch in the response of DRG neurons to ETS transcription factor signaling. PLoS Biol 3:e159. CrossRef Medline

Jones EG, Hendry SHC (1984) Basket cells. In: Cerebral cortex (Peters A, Jones EG, eds), pp 309-336. New York: Plenum.

Kawaguchi Y, Kubota Y (1997) GABAergic cell subtypes and their synaptic connections in rat frontal cortex. Cereb Cortex 7:476-486. CrossRef Medline

Lee S, Hjerling-Leffler J, Zagha E, Fishell G, Rudy B (2010) The largest group of superficial neocortical GABAergic interneurons expresses ionotropic serotonin receptors. The J Neurosci 30:16796-16808. CrossRef Medline

Lee S, Kruglikov I, Huang ZJ, Fishell G, Rudy B (2013) A disinhibitory circuit mediates motor integration in the somatosensory cortex. Nat Neurosci 16:1662-1670. CrossRef Medline

Markov NT, Vezoli J, Chameau P, Falchier A, Quilodran R, Huissoud C, Lamy C, Misery P, Giroud P, Ullman S, Barone P, Dehay C, Knoblauch K, Kennedy H (2014) Anatomy of hierarchy: feedforward and feedback pathways in macaque visual cortex. J Comp Neurol 522:225-259. CrossRef Medline

Meskenaite V (1997) Calretinin-immunoreactive local circuit neurons in area 17 of the cynomolgus monkey, Macaca fascicularis. J Comp Neurol 379:113-132. CrossRef Medline

Miyoshi G, Hjerling-Leffler J, Karayannis T, Sousa VH, Butt SJ, Battiste J, Johnson JE, Machold RP, Fishell G (2010) Genetic fate mapping reveals that the caudal ganglionic eminence produces a large and diverse population of superficial cortical interneurons. J Neurosci 30:1582-1594. CrossRef Medline

Nienborg H, Hasenstaub A, Nauhaus I, Taniguchi H, Huang ZJ, Callaway EM (2013) Contrast dependence and differential contributions from 
somatostatin- and parvalbumin-expressing neurons to spatial integration in mouse V1. J Neurosci 33:11145-11154. CrossRef Medline

Osakada F, Callaway EM (2013) Design and generation of recombinant rabies virus vectors. Nat Protoc 8:1583-1601. CrossRef Medline

Pfeffer CK, Xue M, He M, Huang ZJ, Scanziani M (2013) Inhibition of inhibition in visual cortex: the logic of connections between molecularly distinct interneurons. Nat Neurosci 16:1068-1076. CrossRef Medline

Pi HJ, Hangya B, Kvitsiani D, Sanders JI, Huang ZJ, Kepecs A (2013) Cortical interneurons that specialize in disinhibitory control. Nature 503: 521-524. CrossRef Medline

Seidler B, Schmidt A, Mayr U, Nakhai H, Schmid RM, Schneider G, Saur D (2008) A Cre-loxP-based mouse model for conditional somatic gene expression and knockdown in vivo by using avian retroviral vectors. Proc Natl Acad Sci U S A 105:10137-10142. CrossRef Medline

Sippy T, Lapray D, Crochet S, Petersen CC (2015) Cell-type-specific sensorimotor processing in striatal projection neurons during goal-directed behavior. Neuron 88:298-305. CrossRef Medline

Stryker MP (2014) A neural circuit that controls cortical state, plasticity, and the gain of sensory responses in mouse. Cold Spring Harb Symp Quant Biol 79:1-9. CrossRef Medline

Tamás G, Buhl EH, Lörincz A, Somogyi P (2000) Proximally targeted GABAergic synapses and gap junctions synchronize cortical interneurons. Nat Neurosci 3:366-371. CrossRef Medline

Tamás G, Szabadics J, Lörincz A, Somogyi P (2004) Input and frequencyspecific entrainment of postsynaptic firing by IPSPs of perisomatic or dendritic origin. Eur J Neurosci 20:2681-2690. CrossRef Medline
Taniguchi H, He M, Wu P, Kim S, Paik R, Sugino K, Kvitsani D, Fu Y, Lu J, Lin Y, Miyoshi G, Shima Y, Fishell G, Nelson SB, Huang ZJ (2011) A resource of Cre driver lines for genetic targeting of GABAergic neurons in cerebral cortex. Neuron 71:995-1013. CrossRef Medline

Wall NR, Wickersham IR, Cetin A, De La Parra M, Callaway EM (2010) Monosynaptic circuit tracing in vivo through Cre-dependent targeting and complementation of modified rabies virus. Proc Natl Acad Sci U S A 107:21848-21853. CrossRef Medline

Wall NR, De La Parra M, Callaway EM, Kreitzer AC (2013) Differential innervation of direct- and indirect-pathway striatal projection neurons. Neuron 79:347-360. CrossRef Medline

Wickersham IR, Lyon DC, Barnard RJ, Mori T, Finke S, Conzelmann KK, Young JA, Callaway EM (2007) Monosynaptic restriction of transsynaptic tracing from single, genetically targeted neurons. Neuron 53: 639-647. CrossRef Medline

Xu X, Callaway EM (2009) Laminar specificity of functional input to distinct types of inhibitory cortical neurons. J Neurosci 29:70-85. CrossRef Medline

Xu X, Roby KD, Callaway EM (2010) Immunochemical characterization of inhibitory mouse cortical neurons: three chemically distinct classes of inhibitory cells. J Comp Neurol 518:389-404. CrossRef Medline

Zhang S, Xu M, Kamigaki T, Hoang Do JP, Chang WC, Jenvay S, Miyamichi K, Luo L, Dan Y (2014) Selective attention. Long-range and local circuits for top-down modulation of visual cortex processing. Science 345: 660-665. CrossRef Medline 